\title{
Nonlinear Amplitude Evolution During Spontaneous Patterning of
} Ion-Bombarded Si(001)

\author{
Jonah Erlebacher, Michael J. Aziz \\ Harvard University, Division of Engineering and Applied Sciences, Cambridge, MA
}

Eric Chason, Michael B. Sinclair, Jerrold A. Floro

Sandia National Laboratories, Albuquerque, NM

\begin{abstract}
The time evolution of the amplitude of periodic nanoscale ripple patterns formed on $\mathrm{Ar}^{+}$sputtered Si(001) surfaces was examined using a recently developed in situ spectroscopic technique. At sufficiently long times, we find that the amplitude does not continue to grow exponentially as predicted by the standard Bradley-Harper sputter rippling model. In accounting for this discrepancy, we rule out effects related to the concentration of mobile species, high surface curvature, surface energy anisotropy, and ion-surface interactions. We observe that for all wavelengths the amplitude ceases to grow when the width of the topmost terrace of the ripples is reduced to approximately 25 nm. This observation suggests that a short circuit relaxation mechanism limits amplitude growth. A strategy for influencing the ultimate ripple amplitude is discussed.
\end{abstract}




\section{DISCLAIMER}

Portions of this document may be illegible in electronic image products. Images are produced from the best available original document. 
Spontaneous formation of rippled patterns on surfaces undergoing low-energy ion bombardment $(500-1000 \mathrm{eV})$ is caused by a competition between surface roughening due to the ion beam and surface relaxation via, e.g., diffusion or viscous flow. In principle, sputter rippling should be observable in any material, providing a general probe of surface dynamics. Rippling has been observed in amorphous materials $\left(\mathrm{SiO}_{2}[1]\right)$, metals ( $\mathrm{Ag}[2])$ and semiconductors (Ge [3], Si [4], and others). In some cases, these features are nuisances, such as in sample thinning for transmission microscopy or depth profiling by secondary ion mass spectroscopy. However, sputter rippling also holds potential practical application in nano-scale texturing of optical devices such as solar cells and nano-patterned templates for heteroepitaxial growth. In the microelectronics context, the study of how low energy ion beams interact with surfaces is of great importance in the manufacture of shallow junctions.

Previously, we reported the experimental conditions under which ripples could be produced on $\mathrm{Si}(001)$ (p-type, 3-10 $\Omega$-cm) under $750 \mathrm{eV} \mathrm{Ar}{ }^{+}$bombardment [5]. The cogent experimental details are sputtering a heated sample (between 400 and $700{ }^{\circ} \mathrm{C}$ ) near $70^{\circ}$ from normal (projected along the [110] direction) at high ion flux (of order 0.7 $\mathrm{mA} / \mathrm{cm}^{2}$ ). This procedure yielded rippled samples with wavelength from 200 to $800 \mathrm{~nm}$, depending on the sample temperature. The wavevector of the ripples is perpendicular to the projected ion beam direction. Figure 1 shows an example of a sputter rippled $\operatorname{Si}(001)$ surface prepared in this manner. By measuring the variation of the ripple wavelength with sample temperature and ion beam flux, we determined that the migration energy of the mobile species involved in mass transport (probably addimers) was $1.2 \pm 0.1 \mathrm{eV}$. Also, we determined that the concentration of mobile species was independent of both 
temperature and ion beam flux. We accounted for this behavior with a model in which the concentration is limited by direct addimer removal from the ion beam. In this case, the concentration saturates at a value equal to $Y_{1} / \sigma_{C}$, where $Y_{1}$ is the number of addimers created per incident ion and where the cross-section $\sigma_{\mathrm{C}}$ is related to the area at the surface over which an ion's damage is deposited in the collision cascade. An impinging ion "resets" a surface patch of area $\sigma_{C}$ to an addimer concentration that is independent of $T$ and of its value before the collision.

Here, we report the time evolution of the ripple amplitude and its variation with sample temperature. In particular, we observe that while the ripple amplitude evolves quickly at short times, the amplitude growth always slows down, and ripples eventually reach a steady state amplitude. This result is notable in its contrast to the prediction of standard rippling theory, due to Bradley and Harper (BH) [6]. BH is successful in predicting the relationship between the ripple wavelength, sample temperature and ion beam flux [5] but is formally applicable only at short growth times. In fact, $\mathrm{BH}$ predict that the ripple amplitude grows exponentially at all times, in contrast to our results. As rippling appears in the applied contexts of SIMS and other techniques employing a form of ion milling, the factors that limit amplitude evolution are important to study.

We monitored the time evolution of ripple growth using a novel spectroscopic technique ("Light Scattering Spectroscopy," or LiSSp) whereby the power spectral density (PSD) of the surface was monitored in situ and in real time by broadband UV light scattering and collection by a solid-state spectrometer [7]. The amplitude of features at a particular spatial frequency is proportional to the square root of the PSD at that spatial frequency. The proportionality was found by calibration to the PSD of a 
topograph measured by ex situ atomic force microscopy. The uncertainty in absolute amplitude measured using this technique is of order $25 \%$, and all reported amplitudes are at the upper end of the range. Further details of LiSSp are described elsewhere [8].

Bradley and Harper $(\mathrm{BH})$ provide a theory of sputter rippling that gives the observed relationship between the measured ripple wavelength and the imposed ion beam flux and sample temperature [6]. Theirs is a linear stability analysis of a surface roughening due to sputtering on one hand and annealing by surface diffusion on the other. The standard BH model predicts exponential amplitude growth at short times or, equivalently, small amplitudes.

Before presenting our results, we summarize the salient points of $\mathrm{BH}$. For an ion beam impinging on a surface at an angle $\theta$, measured from normal, a theory of sputter yield due to Sigmund [9] leads to a roughening rate that is proportional to the curvature of the surface in the direction perpendicular to the ion beam, $\kappa$. In $\mathrm{BH}$, the roughening rate is balanced by a relaxation rate proportional to the second spatial derivative of $\kappa$, as in classical relaxation theory. In the moving reference frame of the sputtered surface, the change in height with time, $\partial h(x, t) / \partial t$, can be written

$$
\frac{\partial h(x, t)}{\partial t}=S \kappa(x, t)+B \frac{\partial^{2}}{\partial s^{2}} \kappa(x, t)
$$

where the derivatives are taken with respect to surface arc length, $s$, measured perpendicular to the beam and the curvature is defined to be positive for convex regions of the solid. Under the near-glancing conditions used in this experiment, the roughening prefactor is given by 


$$
S \equiv-\frac{f a}{n} Y_{0}(\theta) \Gamma_{2}(\theta)
$$

where $f$ is the ion flux, $a$ is the average depth of ion energy deposition, $n$ is the atomic volumetric density, $Y_{0}(\theta)$ is the (incidence angle-dependent) sputter yield, and $\Gamma_{2}(\theta)$ is a coefficient governing the erosion rate dependence on the local surface curvature. The relaxation prefactor is given by [10]

$$
B=\frac{D_{s} C \gamma \Omega^{2}}{k_{B} T}
$$

where $\gamma$ is the surface free energy per area, $T$ is the temperature, $k_{B}$ is Boltzmann's constant, $D_{s}$ is the surface diffusivity, $\Omega$ is the atomic volume and $C$ is the concentration (number/area) of mobile species that participate in surface diffusion. Eq. (1) is generally valid when $a K \ll 1$, which was always satisfied in our experiments. Values for the materials constants in Eqs. (2) and (3) are given in the Appendix to this chapter.

Figure 2 shows the ripple amplitude vs. time for samples sputtered under the same ion beam conditions, changing only temperature. The general features of the amplitude evolution are (1) the amplitude evolves steeply at short times, eventually turning over; (2) the shorter the ripple wavelength, the lower the steady-state amplitude; (3) the shorter the ripple wavelength, the faster amplitude saturation is reached.

The BH model of sputter rippling can be analytically solved only in the small slope approximation $\left(\kappa \approx-\partial^{2} h / \partial x^{2}\right)$, valid at short times and small amplitudes. With this 
approximation, Eq. (1) is linear and is solved in Fourier space, predicting exponential increase in time for the amplitudes, $h_{q}(t)$, of modes with spatial frequency $q$ :

$h_{q}(t)=h_{q}(0) \exp \left(R_{q} t\right)$. Experimentally, we clearly do not observe exponential growth at all times. However, aspects of the small slope solution to Eq. (1) are still apparent. For instance, one spatial frequency,

$$
q^{*}=\sqrt{S / 2 B},
$$

grows faster than all others, setting the ripple wavelength, $\lambda^{*}=2 \pi / q^{*}$. In earlier work, we showed how the ripple wavelength scales with temperature and ion flux, experimentally verifying the relationship in Eq. (4). In the regime where the small slope approximation is valid, the amplitude of the ripple, $h_{q^{*}}(t)$, should grow exponentially with rate constant $R_{q^{*}}=S^{2} / 4 B$. In another model for sputter rippling [1], noise due to the stochastic nature of the ion beam was added to the profile evolution equation, Eq. (1). However, this model also employed the small slope approximation and also predicted exponential growth at long times with the same time constant.

Abandoning the small slope approximation in the solution of the BH model, Eq. (1), is the obvious first step in trying to understand why experimentally measured amplitudes do not continue to rise exponentially. The curvature of the surface at any point is exactly given by

$$
\kappa(x, t)=-\left(\partial^{2} H(x, t) / \partial x^{2}\right) /\left(1+(\partial H(x, t) / \partial x)^{2}\right)^{3 / 2}
$$


where $H(x, t)$ is the absolute height profile, related to the height profile in the moving frame of the average ripple position by the transformation

$$
h(x, t)=H(x, t)+f Y_{0} \Omega t .
$$

In terms of $H, \mathrm{Eq} .(1)$ is written

$$
\frac{\partial H(x, t)}{\partial t}=-f Y_{1} \Omega+S K(x, t)+B \frac{\partial^{2}}{\partial s^{2}} \kappa(x, t) .
$$

Eq. (7) can be expressed in a form that can be solved numerically by making the following transformation to dimensionless variables: $y \equiv x q^{*}, \tau \equiv t R_{q^{*}}$, $z(y, \tau) \equiv\left(H(x, t)+f Y_{0} \Omega t\right) q^{*}=h(x, t) q^{*}$. To make this transformation, it is convenient to use the relation, $\partial / \partial s=(\partial x / \partial s) \partial / \partial x$. In terms of the new variables,

$$
\begin{aligned}
\frac{\partial z}{\partial \tau}=-2 \frac{\partial}{\partial y} & {\left[\frac{\partial z}{\partial y}\left(1+\left(\frac{\partial z}{\partial y}\right)^{2}\right)^{-1 / 2}\right] } \\
& -\frac{\partial}{\partial y}\left\{\left(1+\left(\frac{\partial z}{\partial y}\right)^{2}\right)^{-1 / 2} \frac{\partial^{2}}{\partial y^{2}}\left[\frac{\partial z}{\partial y}\left(1+\left(\frac{\partial z}{\partial y}\right)^{2}\right)^{-1 / 2}\right]\right\}
\end{aligned}
$$

Eq. (8) is of a form examined by Srolovitz, et al. [11], albeit in the different context of columnar grain thin film growth. They showed that Eq. (8) produces a ripple that grows 
with spatial frequency $q^{*}=\sqrt{S / 2 B}$, as in the small-slope approximation. Also, as the amplitude of the ripple grows, this wavelength does not change. Furthermore, there is an asymptotic solution in which the amplitude no longer changes significantly with time.

The solution turns out to be roughly sinusoidal, but with somewhat more sharply sloping sides. We have performed our own numerical solution to Eq. (8) to verify this result.

Figure 3 shows our numerical solution of the amplitude evolution, Eq. (8), compared with the amplitude evolution data, plotted in terms of the dimensionless variables, $z, \tau$, introduced above. Our results are clearly inconsistent with the numerical solution of Eq. (8). There are two aspects to this inconsistency. Firstly, and most strongly, the reexpression of our data in terms of the dimensionless variables should have resulted in their "data collapse" onto a universal curve. That is, plotting $h_{q^{*}}(t) q^{*}$ vs. $t \cdot\left(S^{2} / 4 B\right)$ (equivalently, $z_{q^{*}}(\tau)$ vs. $\tau$ ) should have fallen onto the solution of Eq. (8). Clearly, this is not the case. Secondly, the experimentally observed amplitudes saturate at significantly lower values than that predicted by Eq. (8). Alternatively phrased, the amplitude solved numerically continues to rise exponentially to over an order of magnitude higher than that observed experimentally. We have calculated numerical solutions for variants of Eq. (8), in which we added effects of (1) stochastic noise to the initial conditions (i.e., initially, a random profile with specified roughness rather than a flat profile), and (2) stochastic background noise during the numerical amplitude evolution. The same qualitative behavior - that the exponential growth slows at amplitudes higher than seen experimentally - was seen for both variations.

We identify four possibilities for the anomalous low amplitude saturation we observe, only the last one of which we believe to be a viable explanation: (1) the concentration of 
adatoms is changing with time, (2) the surface free energy per area along the sides of the ripples is changing quickly as the amplitude grows due to surface energy anisotropy, (3) diffusivity on the surface is enhanced as the result of peculiar ion-surface interactions, and (4) when the amplitude evolves to a particular height, a faster relaxation mechanism (a "short circuit" mechanism) becomes operative. We discuss each in turn.

1. Concentration Effect. If the concentration, $C$, in Eq. (3), is growing with time, then $B$ will increase, an effect that will tend to damp the "universal" amplitude. There are two reasons to dismiss this effect. Firstly, if $C$ is changing with time, then the ripple wavelength will also be changing with time (through Eq. (4)), an effect we do not observe. Secondly, by analyzing the temperature and flux dependence of the ripple wavelength, we showed that the concentration of adatoms on the surface during rippling should be dependent only on the ratio of the number of adatoms created per incident ion, $Y_{1}$, to the cross-section, $\sigma_{\mathrm{C}}$, over which an ion's damage is deposited in the collision cascade. The sputter yield is related to the angle between the ion beam and the surface normal, which, in principle, changes locally as the amplitude grows. However, because the wavevector of the ripples produced is perpendicular to the ion beam, and the slopes of the ripples never became higher than $32^{\circ}$ (calculated assuming sinusoidal ripples), the angle between the ion beam and the local surface normal changed only at most about $3^{\circ}$ over the course of the experiment. This is a small variation and one calculated assuming the surface could be treated as a continuum. In reality, the surface of the ripples are comprised of steps and terraces, and $Y_{1}$ is likely dependent only on the 
angle between the terraces and the ion beam, which remained unchanged during the course of the experiments.

2. Surface Energy Effect. In principle, the surface free energy per area, $\gamma$, in Eq. (3) is a function of orientation. Because as the ripple grows we are moving from the singular (001) orientation, $\gamma$ will tend to increase. As in scenario \#1, this in turn will tend to increase $B$ and thus damp the "universal" amplitude. But a significantly changing effective gamma will also tend to change the ripple wavelength, which we do not observe.

3. Enhanced Diffusivity. Makeev and Barabasi have demonstrated a number of ionsurface interactions that lead to an apparent enhanced diffusivity, i.e., enhancements to the $S$ term in Eq. (4). One reason for an enhanced diffusivity that they discuss is due to taking the small slope approximation out to higher order terms in the roughening term of Eq. (1) [12]. However, we showed above that taking the full curvature into effect is insufficierit to explain our results.

In a separate paper, Makeev and Barabasi [13] show how a stochastically rough surface can also produce a seemingly enhanced diffusivity. The relevant length scale for their analysis is the scale of the ion implant depth, $L$. By "stochastically rough" they mean that the rms roughness measured over a patch of area equal to $L^{2}$ is of order $L$. We can get a feeling for the rms roughness in our system by estimating the concentration of mobile species on the surface. As shown in the appendix at the end of this chapter, the concentration of addimers on our surface is approximately $C=0.07$ dimers $/ \mathrm{nm}^{2}$, about 
$4 \%$ coverage, or, about 1 addimer in every $14 \mathrm{~nm}^{2}$ patch. Using the Si(001) step height of $0.14 \mathrm{~nm}$, and this value for the concentration, the rms roughness can be estimated to be of order $0.0001 \mathrm{~nm}$. The range of ion damage into the bulk is found using the Monte Carlo code TRIM, and is found to be about $2.9 \mathrm{~nm}$, and thus the surface roughness in our experiment does not satisfy the high roughness conditions mandated by Makeev and Barabasi.

4. Short Circuit Mechanism. In the literature of surface relaxation studies, there have been many examinations of how a sinusoidal profile equilibrates and becomes flat. In a simple model, sinusoidally perturbed crystalline surfaces below their thermodynamic roughening transition temperature relax when like-sign steps along the slopes of the ripple push away from each other, either entropically or through elastic interaction [14]. However, if the amplitude is great enough, then the topmost terrace on the ripple is extremely narrow and a short circuit relaxation mechanism can circumvent step-step interaction.

Two short circuit mechanisms are often discussed. Firstly, the geometry of the surface may be composed of "crossing steps" - like sign steps with very high curvature. When ripples comprised of crossing steps grow to high amplitude, 1-d step curvature at the step extrema drives mass transport, rather than 2-d surface curvature [15]. The second short circuit mechanism is referred to as "terrace pinch-off" $[16,17]$. In this scenario, as the topmost steps get close enough to each other, the steps bounding the extremal terraces can wander into each other (due to thermal fluctuation), and pinch off. Once pinched off, the line tension of the newly-created island perimeters drives 
relaxation. This happens on time scales much faster than the overall relaxation mechanism. A schematic illustrating these two scenarios is shown in Figure 4.

There are three pieces of qualitative evidence we use to support the terrace pinch-off hypothesis. Firstly, a systematic array of crossing steps requires significant miscut [15]. We measured our miscut to be only $0.1 \pm 0.1^{\circ}$, and so the basic geometric criteria may not be met. The [110] ridge direction is consistent with arrays of parallel non-crossing [110]-oriented steps. Secondly, the micrograph in Figure 1 looks bumpy, as in Figure 4A - a characteristic seen in many our micrographs. For the sample in Figure 1, we measure a bumpiness along the ridge of a ripple characterized by a length of about $150 \mathrm{~nm}$. Thirdly, assuming a roughly sinusoidal shape for the ripple, the topmost terrace width can be calculated using the Si(001) step height of $0.14 \mathrm{~nm}$. Figure 5 shows how the width of the topmost terrace never gets smaller than $30 \mathrm{~nm}$ for the conditions used in our experiment and in all cases seems to be approaching a limiting size.

Quantitatively, the length scales predicted by pinch-off model are close to the measured bumpiness. We make a crude estimate for the bumpiness along the ridge by following Pimpinelli, et al. [18], Bartelt, et al. [19], and Erlebacher and Aziz [16], who derived expressions for the pinch-off time when step motion is diffusion limited. The time, $\tau_{P}$ for a fluctuation of length $\lambda_{1}$ and amplitude $A_{\| 1}$ to form (see Figure 5A), is

$$
\tau_{P} \approx \frac{\lambda_{\mathrm{q}} A_{\mathrm{b}}^{2} b^{-3}}{D_{s} C}
$$


where $b$ is the surface lattice spacing of the mobile species. Eq. (9) is expected to be correct to within a geometric factor of order unity. In this system, dimers are mobile, so $b$ is approximately the spacing of the dimer rows, $0.77 \mathrm{~nm}$. We are interested in what amplitude of step fluctuation can occur within the time for the ripples to grow one step height. From Figure 2, the maximum growth rate of the ripples is about $0.002 \mathrm{~nm} / \mathrm{sec}$ and because the step height on $\mathrm{Si}(001)$ is $0.14 \mathrm{~nm}$, the available time for a fluctuation to reach across the topmost terrace is approximately $70 \mathrm{sec}$. For the sample whose AFM micrograph is illustrated in Figure 1, we find $\lambda_{1} A_{\|}^{2} \approx 2.5 \times 10^{5} \mathrm{~nm}^{3}$, using $v=10^{13.2} \mathrm{sec}^{-1}$ as the hopping frequency (see the Appendix) and $C^{\prime}=4 \%$. If the fluctuation is to reach across the top terrace, then $A_{\|}=30 \mathrm{~nm}$, one finds using this crude estimate that $\lambda_{\sharp} \approx 275$ $\mathrm{nm}$, surprisingly (and perhaps coincidentally) close to the measured mound spacing along the top of the ripples seen in the figure $(\approx 150 \mathrm{~nm})$.

In principle, if terrace pinch-off limits amplitude growth, then a smaller growth rate means a greater time available for fluctuations to reach across the topmost terrace. In this scenario, the topmost terrace width would saturate at a larger value than shown in Figure 5 (and the amplitude would be commensurately smaller). We performed a number of runs at lower ion beam fluxes. Unfortunately, the growth rates were so slow that the top terrace width never fully leveled off before the runs were stopped. Qualitatively, however, it appears that they might be saturating at a larger terrace width.

In practical contexts such as depth profiling, rippling is often observed but unwanted. Our results suggest a strategy for avoiding large ripple amplitudes. The saturation in aspect ratio associated with a minimum tenable extremal terrace width implies that 
minimizing the wavelength is an effective way to minimize the ripple amplitude. This should be readily accomplished by minimizing sample temperature.

In summary, we have examined the time evolution of ripple growth on $\mathrm{Si}(001)$ under glancing angle ion bombardment. We find that the amplitude growth does not follow exponential growth predicted using a small-slope approximation in an expression given by Bradley and Harper. Abandoning the small-slope approximation is insufficient to explain our results, as are an enhanced adatom concentration, an effect of surface energy anisotropy, and a stochastically enhanced diffusivity. We propose that the $\mathrm{BH}$ relaxation mechanism is correct only at small amplitudes, and that a short circuit mechanism involving pinch-off of extremal terraces is operative as the amplitude grows.

The authors acknowledge stimulating discussions with Sean Hearne and Craig Arnold, and technical advice given by John Hunter. This work was supported by DEFG08-89ER45401. Portions of this work were performed at Sandia National Laboratories, supported by the United States Department of Energy under contract DEAC04-94AL85000. 


\section{Appendix}

In this appendix, we detail our estimate for the addimer concentration. The experimental parameters necessary to compute $S\left(=-f a \Omega Y_{0}(\theta) \Gamma_{2}(\theta)\right)$ are

$$
\begin{aligned}
& f=0.7 \mathrm{~mA} / \mathrm{cm}^{2}=43.7 \text { ions } /\left(\mathrm{nm}^{2} \mathrm{~s}\right) \\
& a=2.9 \mathrm{~nm} \\
& \Omega=0.02 \mathrm{~nm}^{3} / \text { atom } \\
& \Gamma_{2}\left(\theta=67.5^{\circ}\right)=-0.0634 \\
& Y_{0}\left(\theta=675^{\circ}\right)=0.92 \text { atoms } / \text { ion }
\end{aligned}
$$

yielding $S=0.148 \mathrm{~nm}^{2} / s$.

To compute all factors of $B\left(=D_{s} C \gamma \Omega^{2} / k_{B} T\right)$ except for the concentration, we need to clarify the form of the surface diffusivity, $D_{s}$. Because addimers are diffusing on a square lattice with lattice parameter equal to the dimer-dimer distance, $d=0.77 \mathrm{~nm}$, we can write $D_{S}=(1 / 4) d^{2} v \exp \left(-E_{M} / k_{B} T\right)$, where $v$ is the hopping rate, and $E_{M}$ is the migration energy. For our purposes, the percentage coverage, $C^{\prime}$ of addimers is sufficient.' Using the relation $C^{\prime}=C d^{2}$, we find that $D_{s} C$ can be written $(1 / 4) \vee C^{\prime} \exp \left(-E_{M} / k_{B} T\right)$. The experimental parameters needed for this computation are:

$$
\begin{aligned}
& \gamma=1.23 \mathrm{~J} / \mathrm{m} 2=7.7 \mathrm{eV} / \mathrm{nm}^{2}[20] \\
& \nu=10^{13.2} \mathrm{~Hz} \text { (dimer vibration frequency) [21] } \\
& E_{M}=1.2 \mathrm{eV}
\end{aligned}
$$

yielding $B=C^{\prime} 8.49 \times 10^{3} \mathrm{~nm}^{4} / \mathrm{s}$.

Our measured relationship between the wavelength of the ripple and the ion beam is 


$$
\lambda *(\mathrm{~nm})=\frac{5.18 \times 10^{5} \mathrm{nmeV}^{1 / 2}}{\sqrt{k_{B} T}} \exp \left(-\frac{1.2 \mathrm{eV}}{2 k_{B} T}\right)=2 \pi \sqrt{\frac{2 B}{S}}
$$

Using the above parameters, we find our addimer concentration to be $C^{\prime}=0.04$ atoms $/$ site ( $\approx 4 \%$ coverage), or, $C=0.07$ atoms $/ \mathrm{nm}^{2}$. 


\section{References}

[1] T. M. Mayer, E. Chason, A. J. Howard, J. Appl. Phys 76, 1633 (1994).

[2] S. Rusponi, C. Baragno, U. Valbusa, Phys. Rev. Lett. 78, 2795 (1997).

[3] E. Chason, et al., Phys. Rev. Lett. 72, 3040 (1994).

[4] G. Carter and V. Vishnyakov, Phys. Rev. B 54, 17647 (1996). J. Erlebacher and M. J. Aziz, Mater Res. Soc. Symp. Proc. 440 (1997). Z. X. Jiang and P. F. A. Alkemade, Appl. Phys. Lett. 73, 315 (1998).

[5] J. Erlebacher, M. Aziz, E. Chason, M. Sinclair, J. Floro, Phys. Rev. Lett., in press, 1999.

[6] R. M. Bradley, J. M. E. Harper, J. Vac. Sci. Tech. A 6, 2390 (1988).

[7] E. Chason, M. B. Sinclair, J. A. Floro, J. A. Hunter, R. Q. Hwang, Appl. Phys. Lett. 72, 3276 (1998).

[8] J. Erlebacher, et al., submitted.

[9] P. Sigmund, J. Mater. Sci 8, 1545 (1973).

[10] W. W. Mullins, J. Appl. Phys. 30, 1 (1959). C. Herring, in The Physics of Powder Metallurgy, W. E. Kingston, Ed. (McGraw-Hill, 1951), chap. 8.

[11] D.J. Srolovitz, A. Mazor, B.G. Bukiet, J. Vac. Sci. Technol. A 6 (1988), 2371; A. Mazor, D.J. Srolovitz, P.S. Hagan, B.G. Bukiet, Phys. Rev. Lett. 60 (1988), 424.

[12] M.A. Makeev, A.-L. Barabasi, Appl. Phys. Lett. 71 (1997), 2800.

[13] M.A. Makeev, A.-L. Barabasi, Appl. Phys. Lett. 73 (1998), 1445.

[14] M. Ozdemir, A. Zangwill, Phys. Rev. B 42 (1990) 5013.

[15] H.P. Bonzel, W.W. Mullins, Surf. Sci. 350 (1996), 285. 
Nonlinear Amplitude Evolution During Spontaneous Patterning of Ion-Bombarded Si(001). .

[16] J. Erlebacher and M.J. Aziz, Surf. Sci. 374 (1997) 427.

[17] A. Rettori, J. Villain, J. Phys. France 49 (1988), 257.

[18] A. Pimpinelli, et al., Surf. Sci. 295 (1993) 143.

[19] N.C. Bartelt, J.L. Goldberg, T.L. Einstein, E.D. Williams, Surf. Sci. 273 (1992), 252.

[20] M.E. Keeffe, C.C. Umbach, J.M. Blakely, J. Phys. Chem. Solids 55 (1994) 965.

[21] M. Krueger, B. Borovsky, E. Ganz, Surf. Sci. 385 (1997), 146. 
Nonlinear Amplitude Evolution During Spontaneous Patterning of Ion-Bombarded Si(001).
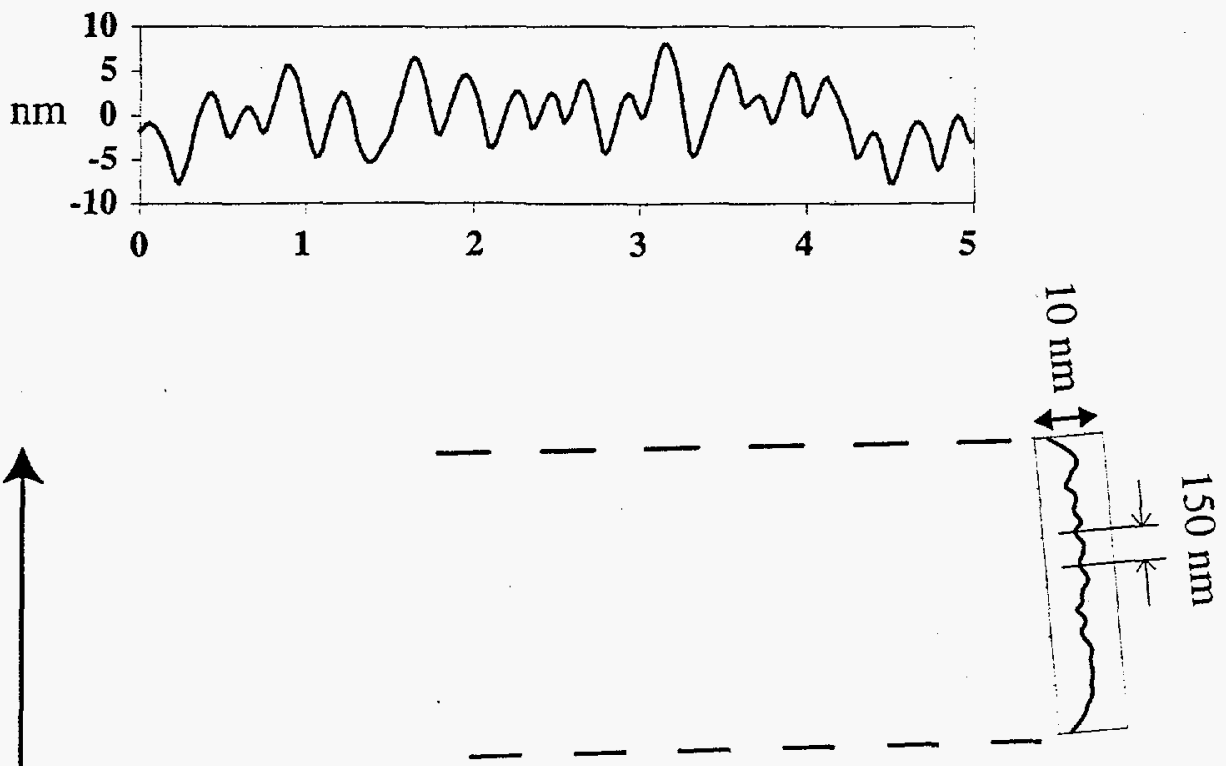

[110]

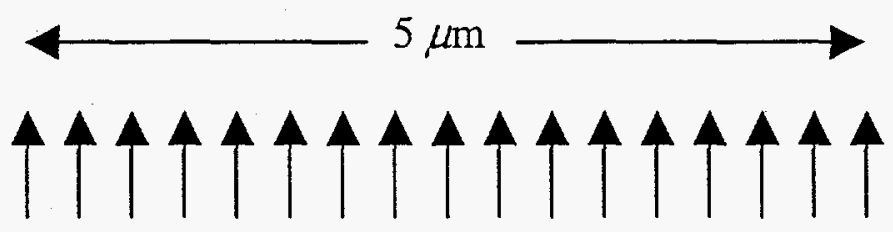

Projected Ion Beam Direction

Figure 1. AFM micrograph of a sputter rippled sample, $\lambda^{*}=360 \mathrm{~nm}, T_{\text {growth }}=555^{\circ} \mathrm{C}$ with height profile through the horizontal centerline and along the ridge of a ripple. 
Nonlinear Amplitude Evolution During Spontaneous Patterning of Ion-Bombarded Si(001).

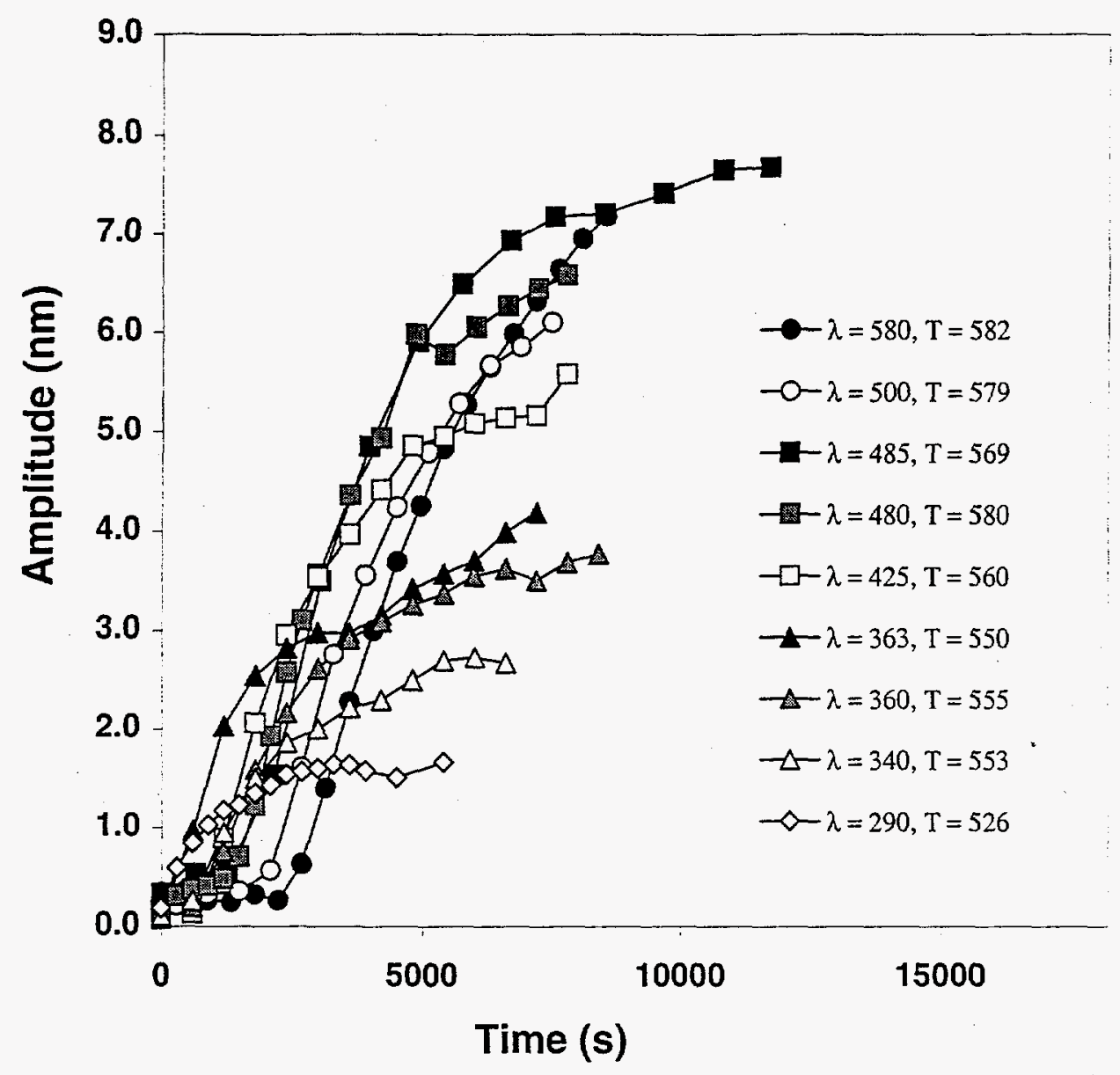

Figure 2. Amplitude ( $\mathrm{nm}$ ) vs. time (s) for sputter rippled samples at different temperatures. Wavelengths are reported in $n m$, and temperatures in ${ }^{\circ} \mathrm{C}$. 


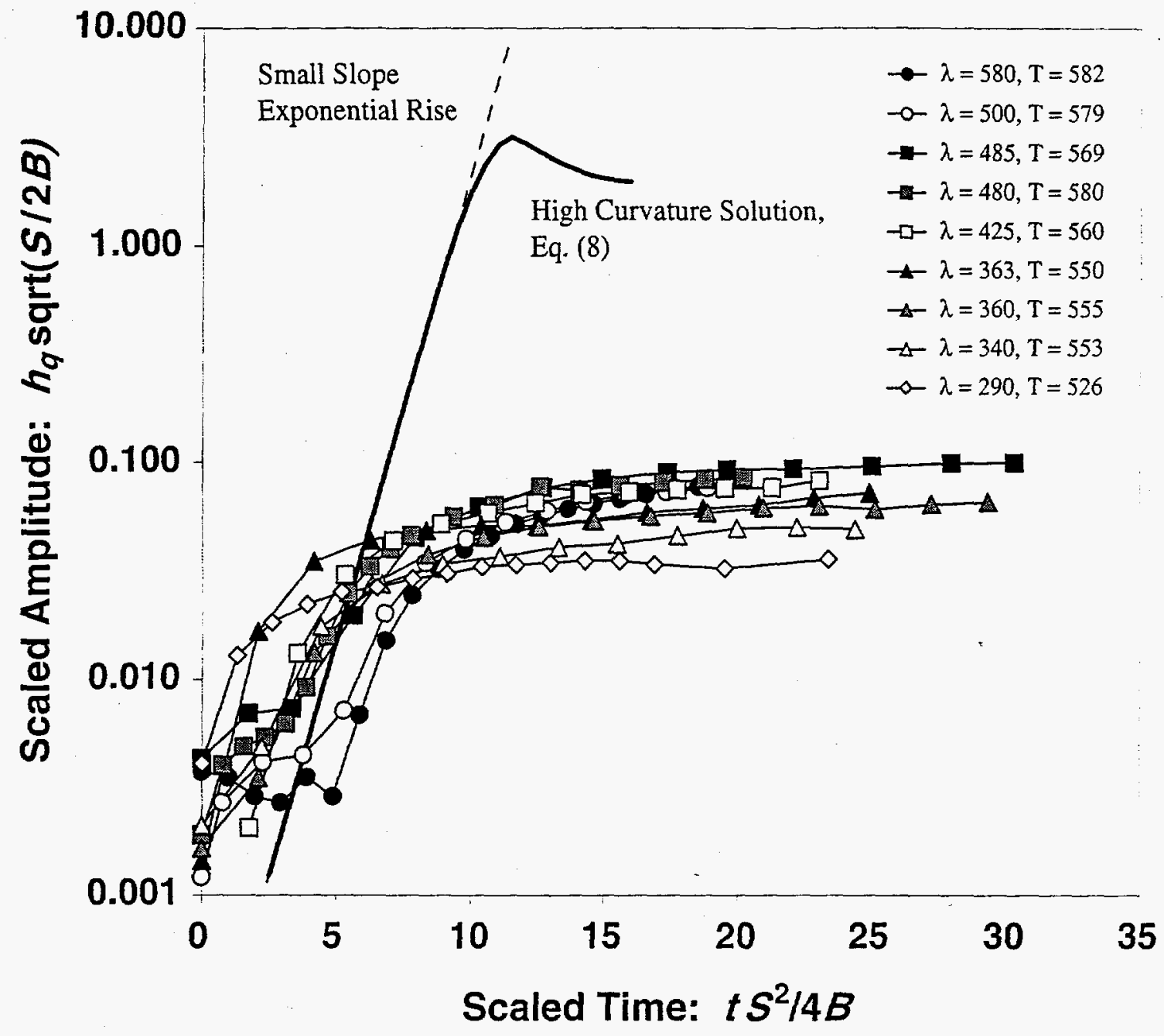

Figure 3. 'Scaled ripple amplitude evolution curves (symbol legend is the same as for Figure 2) vs. time. Also illustrated is the exponential rises predicted by the small slope solution of Eq. (1), and also the numerical solution of Eq. (8) which is equivalent to Eq. (1) but without the small slope approximation. For the numerical and analytical curves, $h_{0}(x)=0.0001$. Ripple wavelengths are reported in $n m$, and temperatures in ${ }^{\circ} \mathrm{C}$. 
Nonlinear Amplitude Evolution During Spontaneous Patterning of Ion-Bombarded Si(001) . .

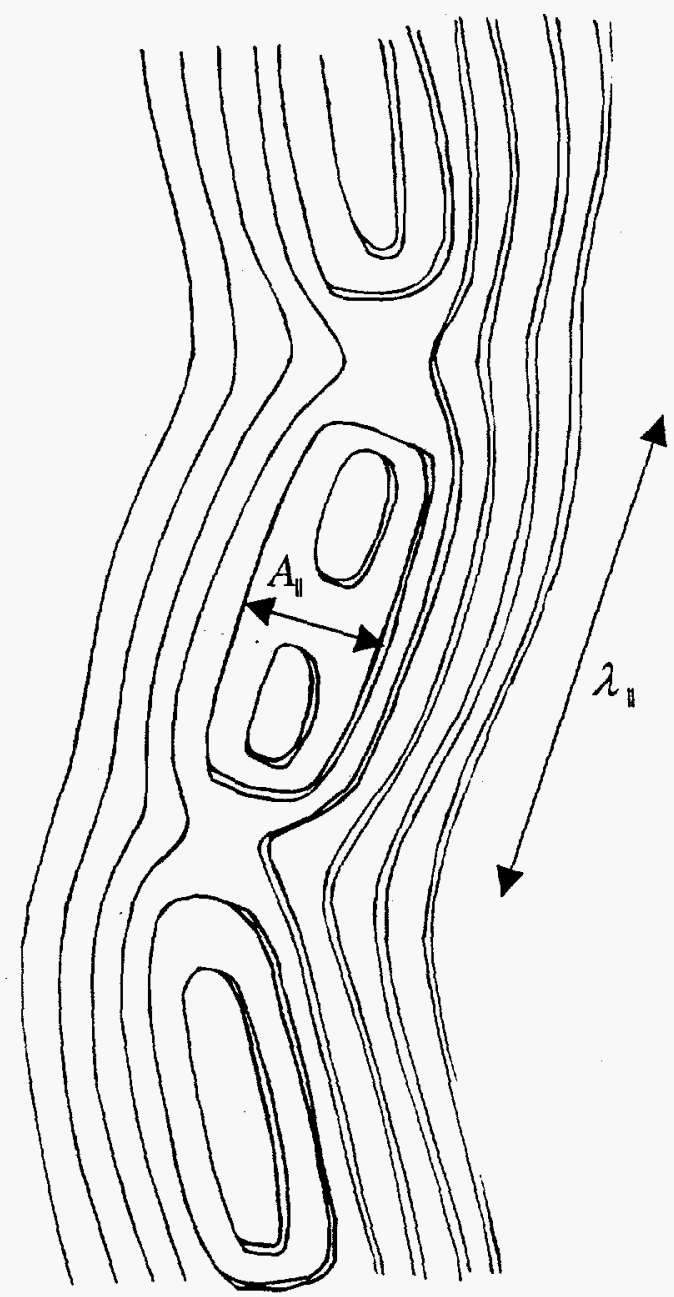

A

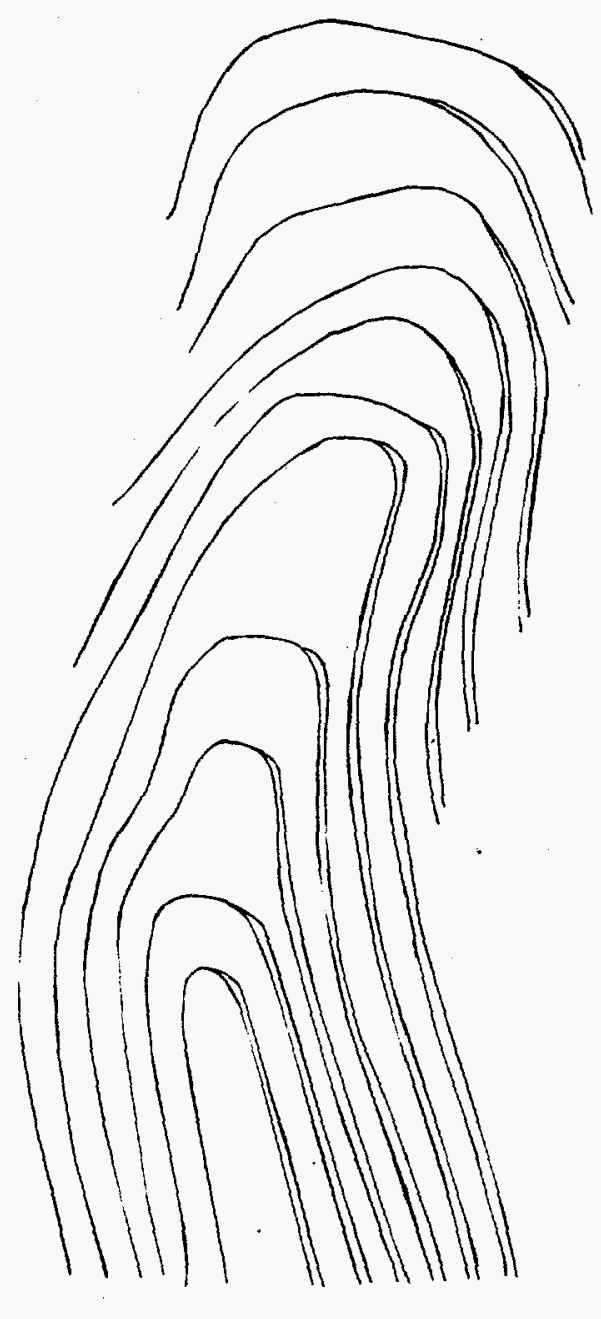

B

Figure 4. Schematic representations of the step structure on rippled surfaces. The real Si(001) surface will have alternating $S_{A}$ and $S_{B}$ steps. (A) Pinched-off terraces at the top of a ripple. The fluctuation that caused this pinch-off has amplitude $A_{\|}$and length $\lambda_{\mathrm{r}}$. (B) High amplitude crossing steps. 
Nonlinear Amplitude Evolution During Spontaneous Patterning of Ion-Bombarded Si(001).

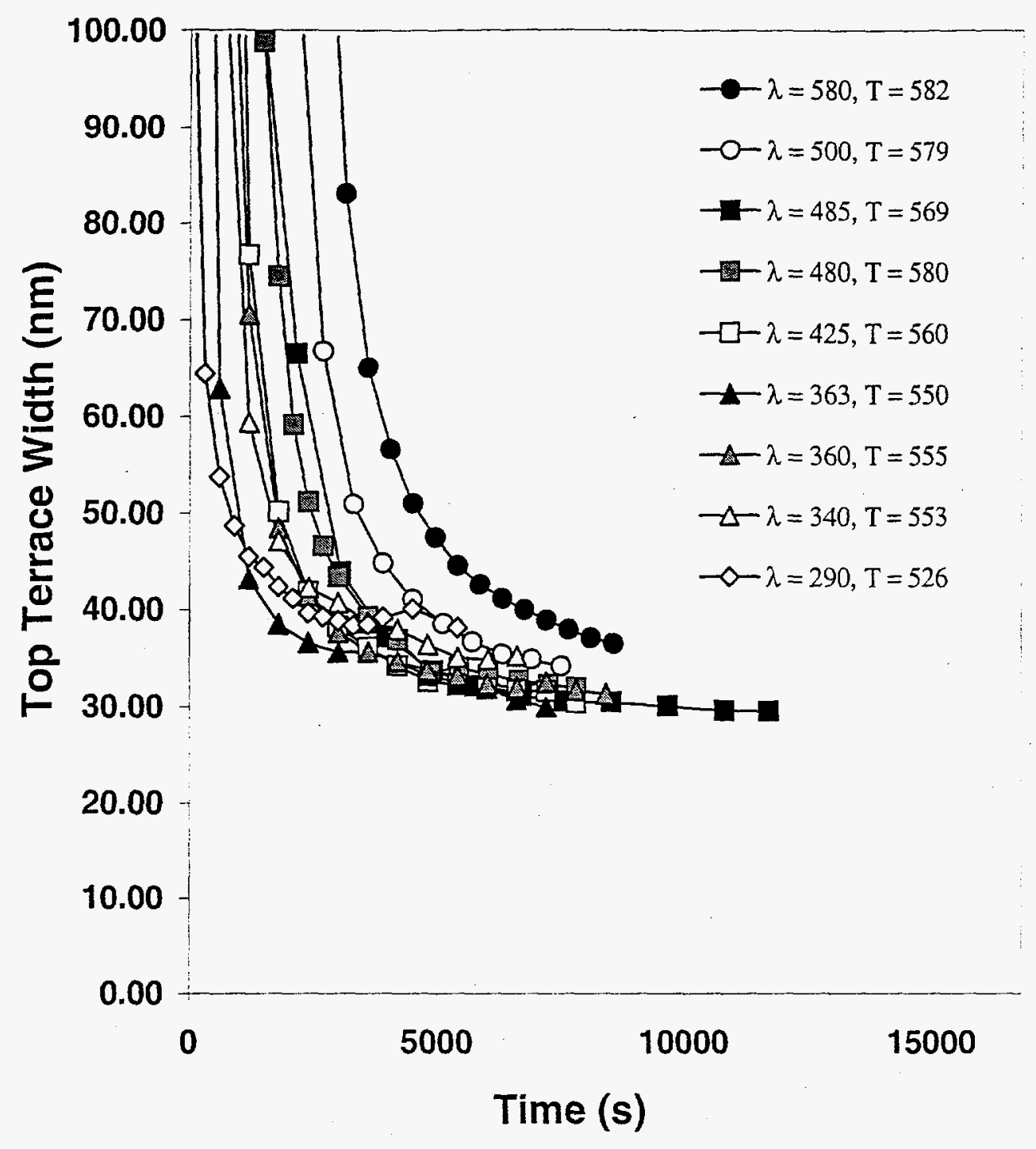

Figure 5. Topmost terrace width versus time for rippled samples at different temperatures. 